\title{
TaskCBP: an intelligent agent for task planning in elderly care
}

\author{
Juan F. de Paz*, Yanira de Paz, \\ Juan M. Corchado and Javier Bajo
}

Departamento Informática y Automática Universidad de Salamanca, Plaza de la Merced s/n, 37008, Salamanca, Spain

E-mail: fcofds@usal.es

E-mail: yanira@usal.es

E-mail: corchado@usal.es

E-mail: jbajope@usal.es

*Corresponding author

\begin{abstract}
This paper presents an autonomous intelligent agent developed for healthcare in geriatric residences. The paper focuses on the role of ambient intelligence in the automation of healthcare services. The work here presented shows the development of an autonomous agent, TaskCBP, which incorporates a model of human thinking, such as reasoning based on past experiences. The planning mechanism integrated within the agent has been implemented by means of a novel QSOR neural network. The system has been tested and this paper presents the results obtained.
\end{abstract}

Keywords: case-based planning; CBP; case-based reasoning; CBR; radio frequency identification; RFID; healthcare; quick self-organising with restrictions neural network; QSOR neural network; ambient intelligence; location system; multiagent systems.

Reference to this paper should be made as follows: de Paz, J.F., de Paz, Y., Corchado, J.M. and Bajo, J. (2010) 'TaskCBP: an intelligent agent for task planning in elderly care', Int. J. Computational Intelligence in Bioinformatics and Systems Biology, Vol. 1, No. 4, pp.349-369.

Biographical notes: Juan F. de Paz is completing his studies of $\mathrm{PhD}$ in Computer Science at University of Salamanca (Spain). He obtained his Technical Engineering in Systems Computer Sciences degree in 2003, Engineering in Computer Sciences degree in 2005 at the University of Salamanca and Statistic in 2007 in the same university. He has been co-author of published papers in several journals, workshops and symposiums.

Yanira de Paz received her PhD from the University of Salamanca (Spain) in January 2008. She obtained her Mathematics degree in 2002 and Statistic degree in 2003 at the University of Salamanca (Spain). She is an Assistant Professor at the Faculty of Economy at the University of Salamanca and co-author of several mathematical and statistical books. She has also been a Lecturer in the Faculty of Mathematics at the Complutense University of Madrid.

Juan M. Corchado received his $\mathrm{PhD}$ in Computer Science from the University of Salamanca in 1998 and $\mathrm{PhD}$ in Artificial Intelligence from the University of Paisley, Glasgow (UK) in 2000. At present, he is an Associate Professor, Director of the BISITE research group (http://bisite.usal.es) and the Director of 
the MSc programs in Computer Science at the University of Salamanca (Spain). Previously, he was Sub-Director of the Computer Science School at the University of Vigo (Spain, 1999-2000) and Researcher at the University of Paisley (UK, 1995-1998). He has been a Research Collaborator with the Plymouth Marine Laboratory (UK) since 1993. He has lead several artificial intelligence research projects sponsored by Spanish and European public and private institutions and has supervised nine $\mathrm{PhD}$. students. He is the co-author of over 150 books, book chapters, journal papers, technical reports, etc. published by organisations such as Elsevier, IEEE, IEE, ACM, AAAI, Springer Verlag, Morgan Kaufmann, etc.

Javier Bajo received his $\mathrm{PhD}$ in Computer Science and Artificial Intelligence from the University of Salamanca in 2007. At present, he is an Associate Professor at the Pontifical University of Salamanca (Spain). He obtained his Information Technology degree at the University of Valladolid (Spain) in 2001 and Engineering in Computer Sciences degree at the Pontifical University of Salamanca in 2003. He has been a member of the organising and scientific committee of several international symposiums such as CAEPIA, IDEAL, HAIS, etc. and co-author of papers published in recognised journals, workshops and symposiums.

\section{Introduction}

During the last three decades, the number of elderly people has risen by about $50 \%$ (Camarinha-Matos and Afsarmanesh, 2002). This sector of the population requires special care and demands novel automated systems oriented to improve their quality of life (Camarinha-Matos and Afsarmanesh, 2002). The paper played by the AmI becomes fundamental in this new scenario since its objectives are directly related to the demands of the elderly people. Ambient intelligence proposes a new way to interact between people and technology. This last one is adapted to individuals and their context, showing a vision where people are surrounded by intelligent interfaces merged in daily life objects (Emiliani and Stephanidis, 2005). AmI creates computing-capable environments with intelligent communication and processing, serving people by means of a simple, natural, and effortless human-system interaction (Richter and Hellenschmidt, 2004). The creation of secure, unobtrusive and adaptable environments for monitoring and optimising elderly people healthcare will become vital. The solutions provided for the AmI should combine hardware devices such as sensors, readers, mobile devices or computers together with novel software solutions. Multiagent systems (Wooldridge and Jennings, 1995) and architectures based on intelligent devices have recently been explored as supervision software systems for medical care for the elderly patients, these intelligent systems aim to support them in all aspects of daily life, predicting potential hazardous situations and delivering physical and cognitive support. Multiagent systems are an evolution of the distributed artificial intelligence (Wooldridge and Jennings, 1995). The application of multiagent systems facilitates taking advantage of their capabilities, such as mobility, pro-activity or social abilities, as well as the possibility of solving problems in a distributed way. Agents, in the context of intelligent environments, must be able to respond to events, take the initiative according to their goals, communicate with other agents, interact with users, and make use of past experiences to find the best ways to 
achieve their objectives. Multiagent systems have become increasingly relevant for developing applications in dynamic, flexible environments, such as personalised user interfaces (Bajo et al., 2006), oceanography (Corchado et al., 2007), tourism (Corchado et al., 2005) or robotic environments (Bajo et al., 2008).

The aim of this paper is to find innovative solutions in the field of ambient intelligence oriented to facilitate the medical care for elderly people. In this way, we present an intelligent agent which incorporates a novel planning system based on the combination of neuronal networks and case-based planning (CBP) systems (Cox et al., 2006). The intelligent agent uses artificial intelligence techniques to improve the working day of the staff in a geriatric residence, which consequently improves the quality of the care provided to the patients. The planning mechanism integrated within the intelligent agent utilises case-based reasoning (CBR) techniques to provide optimum plans (Aamodt and Plaza, 1994). CBR is a paradigm based on the use of past experiences to resolve new problems. When the format of the solution proposed by the CBR system is a sequence of tasks, then we talk about case based planning. CBP allows us to retrieve past experiences when a new plan is created which lends the system a large capacity for learning and adaptation (Cox et al., 2006). The neuronal networks quick self-organising with restrictions (QSOR) proposed within this research framework are self-organised, based on Kohonen (1984) networks, but which present certain improvements. These improvements allow the network to reach a solution much more rapidly. Furthermore, once a solution has been reached, it makes it possible to make new modifications taking restrictions into account (in this study, specifically time restrictions). The new planning mechanism is integrated within autonomous agent for monitoring Alzheimer patients (AGALZ) (Corchado et al., 2006, 2008), a planning agent that works in conjunction with complementary agents into a prototype multi-agent system (ALZ-MAS: Alzheimer multiagent system). The results obtained are compared to those obtained with the previous geodesic planner used by AGALZ.

This work focuses in the development of deliberative agents using a CBP architecture, as a way to implement adaptive systems to improve assistance and healthcare support for elderly and people with disabilities. Agents in this context must be able to respond to events, take the initiative according to their goals, communicate with other agents, interact with users, and make use of past experiences to find the best plans to achieve goals, so we propose the development of a deliberative agent that incorporates a CBP mechanism, specially designed for planning construction. CBP-BDI facilitates learning and adaptation, and therefore, a greater degree of autonomy than that found in pure believe, desire, intention (BDI) architecture (Corchado and Laza, 2003).

In the next section, the elderly residences healthcare problem, together with the possibilities of the ambient intelligence in this kind of environment is presented. In Section 3, the autonomous planner agent proposed in this work is presented. Then, the new planning mechanism is described, finalising with a case study and results and conclusions obtained after the implementation of a prototype into a real scenario.

\section{Ambient intelligence to solve the elderly residences healthcare problem}

One of the most important target groups for domotic systems are the disabled and elderly. There is an ever growing need to supply constant care and support to this section of the 
community (Cesta et al., 2003) and the drive to find more effective ways to provide such care has become a major challenge for Europe (Camarinha-Matos and Afsarmanesh, 2002) and its scientific community (Cesta et al., 2003).

During the last three decades the number of Europeans over 60 years old has risen by about $50 \%$. Today, they represent more than $25 \%$ of the population and it is estimated that in 20 years this percentage will rise to one third of the population, meaning 100 millions of citizens (Camarinha-Matos and Afsarmanesh, 2002). This situation is not exclusive to Europe, since studies in other parts of the world show similar tendencies. In the USA, people over 65 years old are the fastest growing segment of the population (Angulo and Tellez, 2004) and it is expected that in 2020 they will represent about one of six citizens (Camarinha-Matos and Afsarmanesh, 2002) totalling 69 million by 2030. Furthermore, over $20 \%$ of people over 85 years old have a limited capacity for independent living, requiring continuous monitoring and daily care (Angulo and Tellez, 2004).

The importance of developing new and more reliable ways to provide care and support to the elderly is underlined by this trend (Camarinha-Matos and Afsarmanesh, 2002), and the creation of secure, unobtrusive and adaptable environments for monitoring and optimising healthcare will become vital (Angulo and Tellez, 2004). Cesta et al. (2003) consider that tomorrow's healthcare institutions will be equipped with intelligent systems capable of interacting with humans. Multiagent systems and architectures based on intelligent devices have recently been explored as supervision systems for medical care (Angulo and Tellez, 2004) and for the elderly or Alzheimer patients, these intelligent systems aim to support them in all aspects of daily life (Cesta et al., 2003), predicting potential hazardous situations and delivering physical and cognitive support. These systems represent a very sensitive domain, since they deal with data of a critical nature concerning individuals, raising issues of security, and the need to guarantee a highly reliable service that is universally accepted by society (Camarinha-Matos et al., 2004).

The development of distributed multiagent systems requires the use of secure communication networks and identification technology. Wireless fidelity (WiFi) networks with security mechanisms such as WiFi protected access (WPA) offer many possibilities with a reduced infrastructure and low installation costs. Radio frequency identification (RFID) (US Department of Commerce, 2005) is an automated data capture technology that can be used to electronically identify, track, and store information about products, items, components or people. It is most frequently used in industrial/manufacturing, transportation, distribution, and warehousing industries, however, there are other growth sectors including healthcare. The proposed system uses microchips mounted on bracelets worn on the patient's wrist or ankle, and sensors installed over protected zones, with an adjustable capture range up to 2 metres. The microchips or transponders help locate the patients, which can be ascertained by consulting the TaskCBP agents installed in personnel PDAs.

\section{TaskCBP: autonomous agent for healthcare}

Agents can be characterised through their capacities in areas such as autonomy, reactivity, pro-activity, social abilities, reasoning, learning and mobility. These capacities can be modelled in various ways, using different methodologies (Wooldridge and Jennings, 1995). One of the possibilities is to use CBR. The intelligent agent proposed in 
the frame of this research uses CBR to improve its capabilities and generate optimum plans. A CBR system is a dynamic system in which new problems are added continuously to its memory, the similar problems are eliminated and gradually new ones are created by combining several existing ones. This methodology is based on the fact that humans use the knowledge learned in previous experiences to solve present problems. CBR systems record past problem solving experiences and by means of indexing algorithms, retrieve previously stored problems with their solutions (cases) and match and adapt them to a given situation. This means that the set of cases stored in the memory of CBR systems represents the knowledge concerning the domain of the CBR. As discussed below, this knowledge is updated constantly. A typical CBR system, as can be seen in Figure 1, is composed of four sequential steps which are recalled every time a problem needs to be solved (Kolodner, 1993; Aamodt and Plaza, 1994; Watson, 1997):

1 retrieve the most relevant case(s)

2 reuse the case(s) in order to solve the problem

3 revise the proposed solution if necessary

4 retain the new solution as a part of a new case.

Figure 1 Case based reasoning cycle

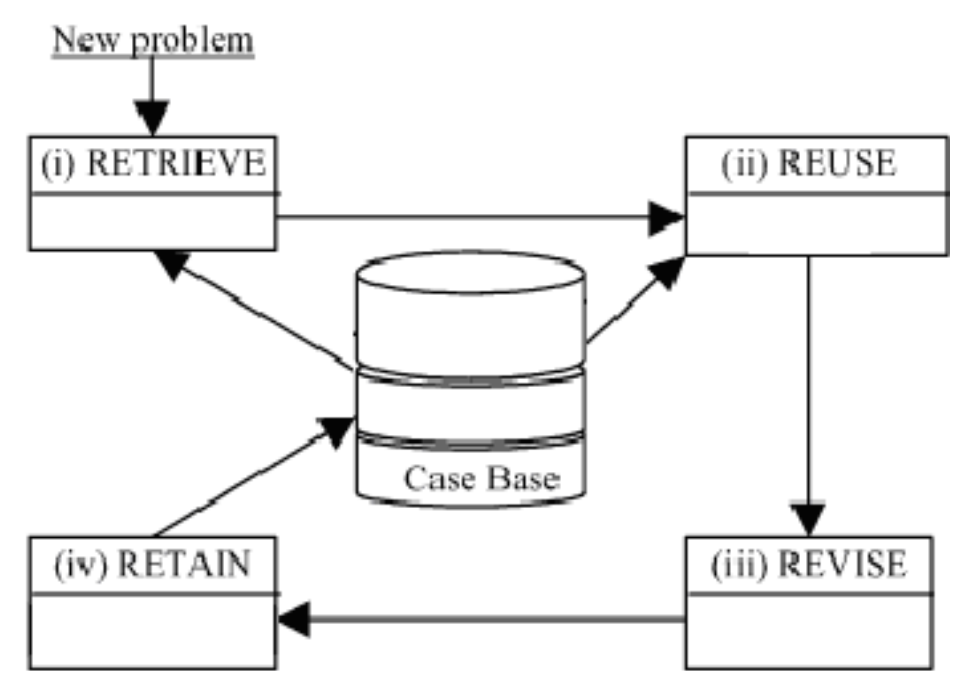

The CBP is a specialisation of CBR based on the generation of plans from cases. The deliberative agents, proposed in the framework of this investigation, use this concept to gain autonomy and improve their guiding capabilities. The relationship between CBP systems and BDI agents can be established by implementing cases as beliefs, intentions and desires which lead to the resolution of the problem. In a CBP-BDI (Corchado and Laza, 2003) agent, each state is considered as a belief; the objective to be reached may also be a belief. The intentions are plans of actions that the agent has to carry out in order to achieve its objectives (Glez-Bedia and Corchado, 2002), so an intention is an ordered set of actions; each change from state to state is made after carrying out an action (the agent remembers the action carried out in the past, when it was in a specified state, and 
the subsequent result). A desire is any of the final states reached in the past (if the agent has to deal with a situation, which is similar to one in the past, it will try to achieve a similar result to the one previously obtained).

TaskCBP is a specialisation of CBP-BDI agents, which incorporates a novel QSOR neural networks to guide the nurses of a residence along their working day: Let $E=\left\{e_{0}, \ldots, e_{n}\right\}$ the set of the possible rooms and places in the residence.

$$
a_{j}: \underset{e_{i}}{E \rightarrow} \rightarrow \underset{j}{\rightarrow}\left(e_{i}\right)=e_{j}
$$

An agent plan is the name given to a sequence of actions (1) that, from a current state $e_{0}$, defines the path of states through which the agent passes in order to offer to the nurse the better path. Below, in (2), the dynamic relationship between the behaviour of the agent and the changes in the environment is modelled. The behaviour of agent $\mathrm{A}$ can be represented by its action function $a_{A}(t) t$, defined as a correspondence between one moment in time $t$ and the action selected by the agent,

$$
\text { Agent } A=\left\{a_{A}(t)\right\}_{t \in T \subseteq N}
$$

From the definition of the action function $a_{A}(t)$, a new relationship that collects the idea of an agent's action plan (3) can be defined,

$$
p_{A}: \underset{\left(t, a_{A}(t)\right) \rightarrow p_{A}(t)}{A}
$$

in the following way,

$$
p_{A}\left(t_{n}\right)=\sum_{i=1}^{n} a_{i A}\left(t_{i}-t_{i-1}\right)
$$

Given the dynamic character that we want to print onto the TaskCBP agent, the continuous extension of the previous expression (4) is proposed as a definition of the agent plan, in other words (5)

$$
p_{A}\left(t_{n}\right)=\int_{t_{0}}^{t_{n}} a_{A}(t) d t
$$

The variation of the agent plan $p_{A}(t)$ will be provoked essentially by: the changes that occur in the environment and that force the initial plan to be modified, and the knowledge from the success and failure of the plans that were used in the past, and which are favoured or punished via learning. The planning is carried out through a neural network based on the Kohonen (1984) network. The neurons are organised in a two layer unidirectional architecture. The learning method is presented as follows: (the equations are presented in the order in that they should be executed).

- $\quad$ To present the input vector $X^{p}=\left(x_{1}^{p}, \ldots, x_{i}^{p}, \ldots, x_{N}^{p}\right)^{T}$ in the input layer.

- The weightings initially take random weightings in $(0,1)$. 
- To calculate the intensity of the neurons of the output layer. The Euclidean distance:

$$
y_{k}=\sqrt{\sum_{i=1}^{N}\left(x_{i}-w_{k i}\right)^{2}}
$$

- To determine the winning neuron that will be that of smaller Euclidean distance.

- To upgrade the weights of the neurons that connect the input layer with the output neuron:

$$
\begin{aligned}
& w_{k i}(t+1)=w_{k i}(t)+\eta(t) g(k, h, t)\left(x_{i}(t)-w_{k i}(t)\right) \\
& g(k, h, t)=e^{\frac{-l k-\left.h\right|^{2}}{2 R(t)^{2}}}
\end{aligned}
$$

(Gaussian function), therefore, the formula is the following:

$$
w_{i}(t+1)=w_{k i}(t)+\eta(t) e^{\frac{-l k-h h^{2}}{2 R(t)^{2}}}\left(x_{i}(t)-w_{k i}(t)\right)
$$

where $w_{k i}$ is the weight of the connection between the input neuron $i$ and the output neuron $k$; $t$ is the iterations; $\eta$ is the learning rate; $h$ is the position of the winning neuron; $k$ is the neuron of the output layer; and $i$ is the neuron of the input layer. In $k-h$ a distance is calculated between the neurons. The Euclidean distance has been used.

\section{QSOR neural networks}

The basic Kohonen (1984) network cannot be used to resolve dynamic problems since it attempts to minimise distances without taking into account any other type of restriction, such as time limits. In the present study, a planner based on Kohonen networks is described but with a number of improvements (QSOR neural network) that allow us to reach a solution more rapidly. Furthermore, once a solution has been reached, it is re-modified in order to take restrictions into account. As such, for this modification of the basic algorithm (QSOR), the aim is to make the solution search more agile and in order to achieve this, the basic vicinity function used in the Kohonen network is modified. The number of neurons in the output layer corresponds to the places that the user (or traveller) wishes to visit. The topology of the neural network being considered is described below. The input layer is formed by two neurons, each one of those receives the coordinates of the place presented as input. A vector of neurons is used of size the same as the number of places to visit in the problem in the output layer, as shown in Jin et al. (2003) and Leung et al. (2004). The number of neurons in the output layer is not modified. Let $x_{i} \equiv\left(x_{i 1}, x_{i 2}\right) i=1, \ldots, N$ the coordinates from the place $i$ and $n_{i} \equiv\left(n_{i 1}, n_{i 2}\right) i=1, \ldots, N$ the coordinates of the neurons $i$ in $\mathfrak{R}^{2} . N$ places will be visited by the traveller. Consequently there will be: two neurons in the input layer and $N$ neurons in the output layer. It will be considered a vicinity function decreasing with the number of iterations. 


$$
g(k, h, t)=\operatorname{Exp}\left[\left(-\frac{|k-h|}{N / 2}\right) \frac{\underset{\substack{i, j \in\{1, \ldots, N\} \\ i \neq j}}{\min }\left\{f_{i j}\right\}-\sqrt{\left(n_{k 1}-n_{h 1}\right)^{2}\left(n_{k 2}-n_{h 2}\right)^{2}}}{\operatorname{Max}_{\substack{i, j \\ i \neq j}}\left\{f_{i j}\right\}}-\lambda \frac{|k-h| t}{\beta N}\right]
$$

$\lambda$ and $\beta$ are determined empirically, their respective values are: five and 50. $t$ is the current iteration. $\operatorname{Exp}[x]=e^{x} . N$ is the number of places that are visited by a traveller and $f_{i j}$ is the distance given by the Floyd algorithm.

The radius of final vicinity should be near to zero so that only the winner is upgraded. Iteratively the group of places will be presented, so that the weights of the neurons approach the coordinates of the places. When concluding the process, there will be a neuron associated to each place. To determine the route to follow, we will leave the place associated to the neuron $i$ to the associated to $i+1$, for $i=1,2, \ldots, N$, passing the whole vector of neurons. To close the road, the last tract will be given by the route that joins the place associated to the neuron $N$ with the associated to the neuron 1 . The distance of the road will be given by the sum of the distances between the successive couples of rooms of the road. The learning rate is a decreasing function as shown in (11):

$$
\eta(t)=\operatorname{Exp}\left[\sqrt[-4]{\frac{t}{\beta N}}\right]
$$

The activation function of the neurons corresponds to the identity function. When the system stops, the route to continue will be given by the weights of the neurons that will be very close to the coordinates of the places. To know which following place to visit, we need to study the following element of the vector of neurons. The neurons are stored in a vector that contains the weights of each neuron at present, so that the vector defines a ring, the neuron $n_{1}$ is the following to the $n_{N}$ considered.

With a big radius of vicinity, in the first iterations of the algorithm the victory of a neuron affects a great part of the map, so that a global self-organisation takes place. If the radius decreases, the effect of a victory affects every time a smaller part of the map, so that the criterion to stop the learning of the network is that the distance among places cannot be optimised more. The initial number of total iterations is of $T_{1}=\beta N$ (first phase). When $t=\beta N$, all the couples of possible neurons are exchanged (exchanging their weights) in the obtained ring of neurons and if the distance is optimised then the learning is not finished. In general, in the phase $Z$, the total number of interactions to carry is (12).

$$
T_{Z}=T_{Z-1}-\frac{T_{Z-1}}{Z}
$$

The aim of these phases is to eliminate the crossings. Concluded the iterations of each phase is proven if the distance is already optimised, in such a way that in the phase that stops the learning, the distance is minimum. The primary objective - to achieve artificial neural networks that are faster than basic Kohonen networks, applied to the problems that the basic networks resolve - has been achieved. In the section below, the necessary modifications are introduced into the algorithm so that the network can take restrictions into account, and therefore, be able to resolve other problems that cannot be resolved by basic Kohonen networks. 
Another interesting improvement to the Kohonen networks consists of taking time restrictions into account. Instead of using the distance calculated by means of the Floyd algorithm, a different distance is used that we call 'temporal distance'. Without loosing any generality it can be supposed that a distance unit is equivalent to a time unit.

$$
d t_{i j} \equiv d t\left(x_{i}, x_{j}\right)=\operatorname{Max}\left(f_{i j}+t_{i}, b_{j}\right)
$$

where $t_{i}$ is the time hended to get to place ' $i$ ' from the previous place plus the time taken on tasks to be carried out within place ' $i$ ' (in other words, the service time in ' $i$ ') and $b_{j}$ is the time limit for carrying out the tasks in place ' $j$ '. In this way, the vicinity function of the network modified from the QSOR network is (14).

$$
\begin{aligned}
& g(k, h, t)=\operatorname{Exp}\left[\left(-\frac{|k-h|}{N / 2}\right) \frac{\underset{\substack{i, j \in\{1, \ldots, N\} \\
i \neq j}}{\operatorname{Max}}\left\{d_{i j}^{*}\right\}-\sqrt{\left(n_{k 1}-n_{h 1}\right)^{2}\left(n_{k 2}-n_{h 2}\right)^{2}}}{\operatorname{Max}_{\substack{i, j \\
i \neq j}} \dot{d}\left\{d_{i j}^{*}\right\}}-\lambda \frac{|k-h| t}{\beta N}\right] \\
& d_{i j}^{*} \equiv d^{*}\left(x_{i}, x_{j}\right)=\frac{f_{i j}+t_{i}}{d t_{i j}}
\end{aligned}
$$

\section{Case study: intelligent environment for elderly residences}

The residence Santísima Trinidad of Salamanca has been interested in improving the services offered to its patients and has collaborated in the development of the technology presented here, providing their know-how and experimenting with the prototype developed. This residence is intended for people over 65 years old, and has the following services and facilities among others: TV room, geriatric bathroom, hairdressing salon, medical service, religious attention, occupational therapy, technical assistance, terrace, garden, laundry service, clothes adjustment, infirmary, reading room, living room, room of visits, cafeteria, social worker, chapel, elevator, customised diet, and multipurpose room.

\subsection{Elderly residence intelligent environment}

The AmI-P system is a distributed system of a relatively high dimension, as shown in Figure 2. Studying the requirements of the problem, four roles have been chosen: the Patient role manages the patient's personal data and behaviour (monitoring, location, daily tasks, and anomalies); the doctor role treats patients; the nurse role schedules the nurse's working day obtaining dynamic plans depending on the tasks needed for each assigned patient; the manager role controls the patients' location and manages locks and alarms; besides, the manager role manages the medical record database and the doctor patient and nurse-patient assignment. The dependences and relations between roles are also described. For the roles involved in the system, a number protocols have been considered: request a treatment, inform about monitoring data, inform about care results, request a doctor assignment, request a nurse assignment, inform about assignment, request a patient's daily plan, inform about a patient's daily tasks, request a patient 
location, inform a nurse about a lock activation, report alarm activation, request doctor situation, doctor reports on his schedule, request a nurse situation, nurse reports situation, patient reports an anomaly, patient reports on personal data and previous medical records. For example, when the nurse wants to know the tasks required for the patient, the nurse role executes a protocol RequestPatientPlanif through which is able to make a request to the patient role. The patient role acts to give a suitable response to the nurse role and executes the InformPlanif protocol to communicate the planned tasks to the nurse role.

Figure 2 AmI-P wireless technology organization schema

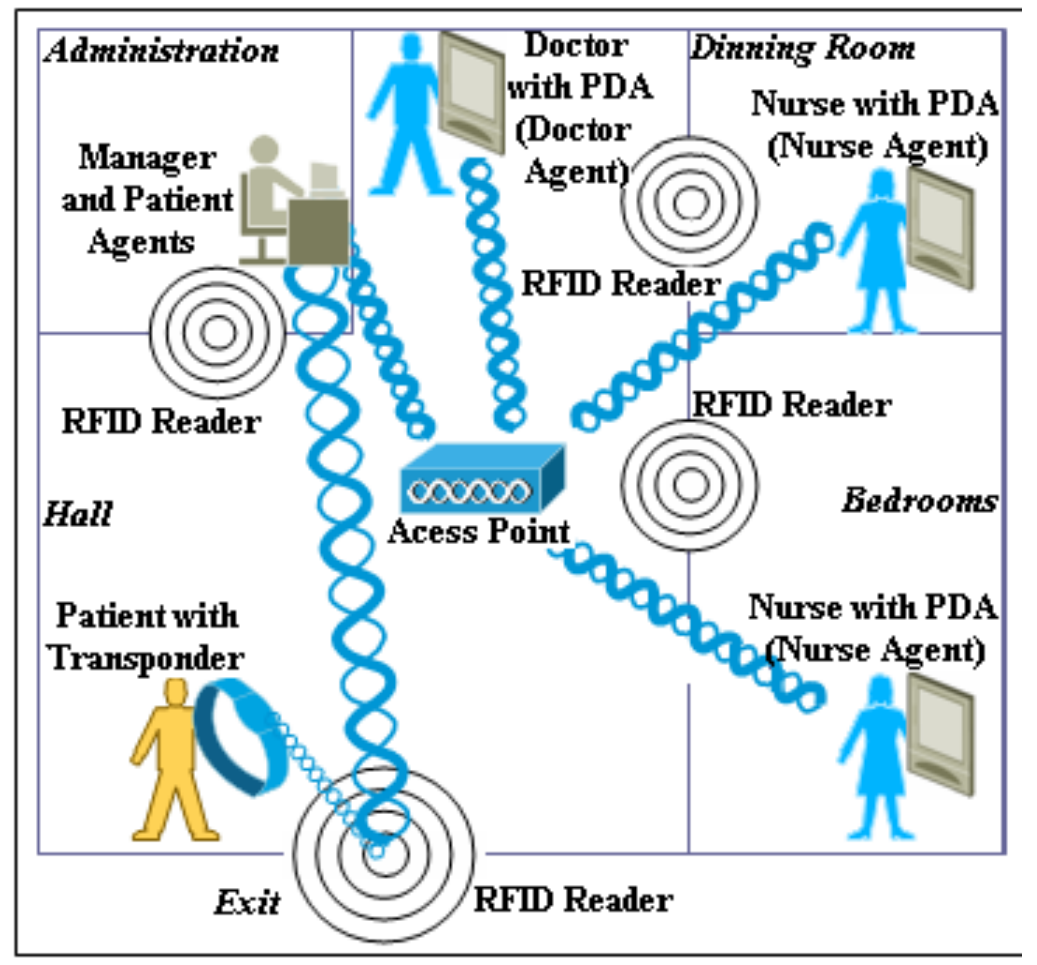

The conclusions obtained after the analysis and design process let us conclude that AmI-P is composed of four different types of roles:

- Patient role manages the patient's personal data and behaviour (monitoring, location, daily tasks, and anomalies). Every hour validates the patient location, monitors the patient state and sends a copy of its memory base (patient state, goals and plans) to the manager role in order to maintain backups. The patient state is instantiated at execution time as a set of objects and these objects are controlled through goals that must be achieved or maintained. The objects that were seen to define a general patient state at the Santísima Trinidad residence of Salamanca were: weight, temperature, blood pressure, feeding (diet characteristics and next time to eat), oral medication, parenteral medication, posture change, toileting, personal hygiene, and exercise. The objects and goals used for every patient depend on the plan (treatment) or plans that the doctors prescribe. The patient role monitors the patient state by means of the goals. To know if a goal has been achieved or has failed, it is necessary 
to maintain continuous communication with the rest of the AmI-P roles. At least once per day, depending on the corresponding treatment, the patient role must contact the nurse role. The patient role must have periodic communication with the doctor role. Finally, the patient role must ensure that all the actions indicated in the treatment are taken out.

- Manager role controls the patients' location and manages locks and alarms as well as the management of the medical record database and the doctor-patient and nurse-patient assignment. It must provide security for the patients and medical staff and the patients, doctors and nurse assignment must be efficient.

- Doctor TaskCBP role treats patients. The doctor role needs to interact with the patient role to order a treatment and receive periodic reports, with the manager role to consult medical records and assigned patients, and with TaskCBP role to ascertain the patient evolution.

- TaskCBP role schedules also the nurse's working day obtaining dynamic plans depending on the tasks needed for each assigned patient. TaskCBP manages nurses' profiles, tasks, available time and resources. The generated plans must guarantee that all the patients assigned to the nurse are given care. The nurse can not exceed eight working hours. Every role generates personalised plans depending on the nurse's profile and working habits.

Manager and patient roles run in a central computer, but TaskCBP roles run on mobile devices, so a robust wireless network has been installed as an extension to the existing wired LAN. With respect to the question of failure recovery, a continuous monitoring of the system is carried out. Every role saves its memory (personal data) onto a database. The most sensitive roles are patient roles, so these roles save their state every hour. When a role fails, another instance can be easily created from the latest backup. The database and server used must have redundancy and failure recovery, so a redundant array of inexpensive disks (RAID) server is used. In the case of a server failure, an alarm is generated and all the plans and information required for nurses and doctors to carry out their working day are automatically printed. A secure and authenticated access to the patient's data is provided. The use of different authorisations for users, logins and passwords, and the encryption of messages using a public key infrastructure and secure socket layer (SSL) have already been implemented. Moreover, the RFID tag only contains the identification number, and not personal data.

Figure 3 shows a diagram of the first floor of the Santísima residence of Salamanca, an institution interested in our technology, containing the main facility rooms, while all the patients' rooms are located in the second floor. This residence has capacity for 60 patients, an average of six nurses, one social worker and five more employees with other responsibilities. We selected 30 patients to test the system, so the hardware implemented at the residence basically consisted of 42 ID door readers (Hitag HT RM401and mobile WorkAbout Pro RFID), one on each door and elevator, four controllers, one at each exit, one in the first floor hall and another in the second floor hall, and 36 bracelets (Sokymat ID Band Unique Q5 with a chip Hitag S 256), one for each patient and the nurses. The ID door readers get the ID number from the bracelets and send the data to the controllers which send a notification to the manager agent. 


\subsection{Tasks planning system}

The objectives of TaskCBP planner agents are: to plan the nurses and doctors working time dynamically, to maintain the standard working reports about their activities, and to guarantee that the patients assigned to the nurses are provided with suitable care. Thus, the TaskCBP planner agent schedules the working days obtaining dynamic plans depending on the tasks needed for each assigned patient.

Figure 3 Sensor positioning in the first floor of the Santísima Trinidad residence of Salamanca

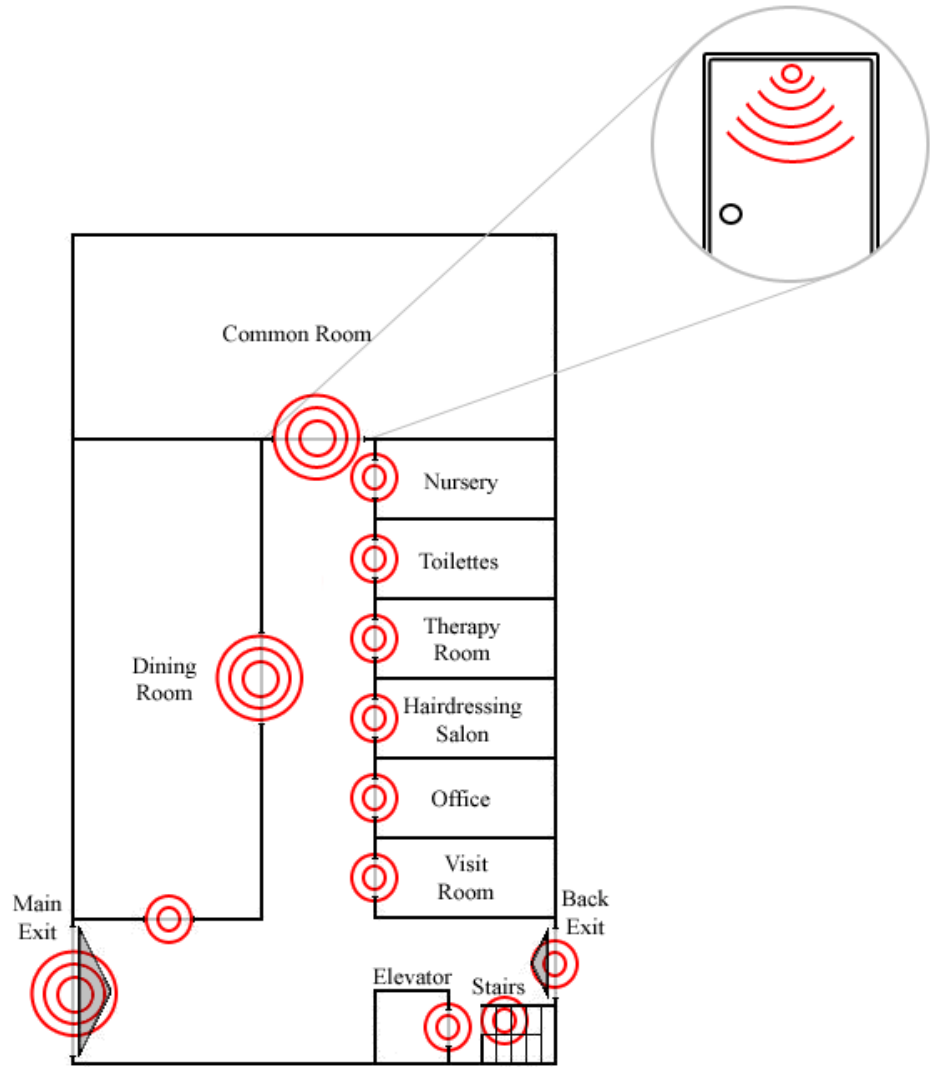

Given a set of memories B compatible with the problem E, it is possible to generate a plan base CBP that contains all the possible plans produced by the combinations of compatible memories. The memories available for the TaskCBP planner agent are tasks, resources and time. A task is a java object that contains the data of the patient who requested the service, the description of the service and the time limits to carry it out. For each task one or more goals are established, in such a way that the whole task is eventually achieved. A goal is also a java object, that identifies what the TaskCBP planner agent wants to achieve (complete a task) and under which conditions (restrictions). For this, a goal can contain parameters and define creation conditions (that allow TaskCBP to define the conditions for achieving the goal), context conditions (the conditions that must be fulfilled) or drop conditions. 
The CBP system constructs plans as a sequence of tasks that need to be carried out by a nurse. A description of the problem will be formed by the tasks that the nurse needs to execute, the resources available, and the times assigned for their shift. In the retrieval stage, the descriptions of similar problems are recovered. In order to do this, the TaskCBP planner agent allows the application of various similar algorithms (cosine, clustering etc.). In this step, those problem descriptions found within a range of similarity close to the original problem description are recovered from the beliefs base. In our case, a tolerance of $20 \%$ has been permitted.

Once the most similar problem descriptions have been recovered, the reuse stage recovers the solutions associated with them. One solution contains all the plans (sequences of tasks) that are carried out in order to achieve the objectives of TaskCBP for a problem description (assuming that replanning is possible) in the past, as well as the efficiency of the solution being supplied. At this time, TaskCBP takes control of the processing of the plan (scheduling). The reuse stage is centred around the objectives and resources needed by each task, as well as on the objectives that the nurse needs to perform and the resources available in order to carry out the global plan. The objectives or global plans that each nurse has are to attend to the patients and not to work for over eight hours. The time available is a problem restriction. Reuse stage takes care of incidents and interruptions that may occur during replanning.

In order to illustrate how the planner works, let's take a significant example. In the first place, it is necessary to take into account that each nurse has a different profile according to their qualification and the tasks that they usually carry out. Let $p r=\left\{p r_{1}, \ldots, p r_{10}\right\} \subset I$ define the stored profiles of the nurses at the residence. It is considered appropriate to manage the profiles of the nurses because there are some nurses who perform tasks with greater skill or who carry out tasks in less time. On the other hand, the TaskCBP planner agent maintains a close relationship with the manager. The manager has as one of its tasks the assignation of nurses to patients and doctors to patients. This assignation is carried out through the CBR reasoning motor of the manager. When the new assignation of tasks needs to be carried out to the nurses or to the doctors, both past experiences, such as the profile of the nurse or doctor, and the needs of the current situation are retrieved. In this way tasks are allocated to a nurse. These tasks may correspond to the same patient or to a number of patients. Moreover, as mentioned above, the profile of each nurse is taken into account. For example, not all nurses are equally qualified for rehabilitation. If one nurse is more qualified in the area, she will be allocated the patients whose need for rehabilitation is greater, always taking into account that the nurse cannot work more than eight hours, so that the number of patients assigned depends on the time needed to carry out the rehabilitation. The manager takes into account how those patients who receive rehabilitation are improving, the arrival of new patients, holiday rotas etc. As such, the allocation of tasks needs to be set on a daily basis.

Secondly, it is necessary to store within the beliefs base the time that each task takes, described as $t_{j}=\underset{j, k}{\operatorname{Max}}\left\{t_{j k}^{i}\right\}$, where $j$ indicates the type of task, $k$, the nurse with the most suitable profile to carry it out (since it is only possible to assign on each task type to the nurses who are qualified to carry it out) and $i$, the patient that requires the task, $t_{j} \subset I$.

The first step when working with CBRs is obtaining a definition of the case structure. A case structure is composed of three elements: a problem description, the solution 
applied to solve the problem and the result obtained for the solutions applied (Bergman et al., 2005). For the current problem the case is described as follows:

- Problem description: Describes the initial information available for generating a plan. The problem description for planning a nurse's working day consists of a case identification, the list of tasks that the nurse has to accomplish, the nurse's profile, the nurse's preferences and temporal restrictions.

- Solution: Describes the actions carried out in order to solve the problem description. A solution contains the case identification, the timetables and the priorities for each task and the route or sequence of tasks.

A solution is composed of a case identification, timetables, and a route. The TimeTables field contains a list of Timetable elements. A Timetable element indicates the identification of a task, the priority of a task, the desired starting and finishing times for a task and the room identification if required. The route field contains a description of the activities carried out in a room of the residence, including rooms visited, arrival and service times, patients and tasks.

- Efficiency: contains the case id and a number indicating the plan efficiency.

For each of the rooms in the residence, the system manages information related to the room location (in coordinates), access (doors) location (in coordinates), room type and resources available.

In our case study, at the beginning of each working day, every nurse receives a list of tasks that must be completed. The tasks must be assigned to the nurses. It is necessary to take into account that each nurse has a different profile according to their qualification and the tasks that they usually carry out. It is considered appropriate to manage the profiles of the nurses because there are some nurses who perform tasks with greater skill or who carry out tasks faster. The structure of a nurse profile shows the abilities of a nurse, her service time and timetable preferences and restrictions.

One of the manager agent's duties is the assignation of nurses to patients. This assignation is carried out through a CBR reasoning motor integrates within the manager Agent. When a new assignation of tasks needs to be carried out to the nurses, both past experiences, such as the nurse profile, and the needs of the current situation are retrieved. In this way, tasks are allocated to a nurse. These tasks may correspond to the same patient or to a number of patients. The task structure is shown in Table 1. Moreover, as mentioned above, the profile of each nurse is taken into account. For example, not all nurses are equally qualified for rehabilitation. If one nurse is more qualified in the area, she will be allocated the patients whose need for rehabilitation are greater, always taking into account that the nurse cannot work more than eight hours, so that the number of patients assigned depends on the time needed to carry out the rehabilitation. The manager agent takes into account how those patients who receive rehabilitation are improving, the arrival of new patients, holiday rotas etc. As such, the allocation of tasks needs to be set on a daily basis.

Once the assignation of tasks to a nurse has been completed, the assignation is communicated to the corresponding Nurse agent. That is, the nurse agent receives a new problem description needing to be resolved. From this moment on, the CBP planning process begins. The nurse agent must take into account the time that the nurse has 
available and the time required for each task. Moreover, the resources available and the location of the patients involved are also taken into account.

Table 1 Task structure

\begin{tabular}{lc}
\hline Task field & Field type \\
\hline TaskData & TaskData \\
ServiceTime & Time \\
Date & Date \\
Room & Room \\
Resources & ArrayList of resource \\
\hline
\end{tabular}

The location of the patients is a factor which significantly influences the decision as to whether a plan should be interrupted. For example, in the case that a nurse should go to a given room to take dinner to the patient and the patient is actually in a different room, the nurse's plan will need to be interrupted. As mentioned above, the location of the patients within the residence is defined through a reference system in $\mathfrak{R}^{2}$. In the location system, it is fundamental that RFID devices are used. These devices make it possible to rapidly assess the possibility or need to replan.

A plan can be interrupted for different reasons. Those which have been taken into account within the residence are: that a resource fails, that a patient suffers some sort of crisis and requires unforeseen attention that the patient has an unexpected visit or that visits to the patient have gone on over the permitted time allowed and an emergency situation. If the planner finds itself in a situation where the plan is interrupted, it rejects the initial plan and seeks an alternative one. The first thing that needs to change is the task order, attempting to maintain the assignation originally allocated by the manager. The new plan must meet the initial objectives. In the event that this is impossible, the nurses will need to be reassigned. This reassignment will attempt to limit changes to a minimum. For reassignment, it is necessary to take into account the tasks that were assigned to the nurses, the development of the plans (which tasks have been carried out and which still need to be done) and the profiles of the nurses (prioritising preparation for the task that cannot be covered). The nurse who is assigned the task should replan in order to include the new task. In the event that the replanning is positive (the tasks that still need to be done and the new task can be carried out) the process is complete. If the replanning is negative, the next nurse down in the ranking will be used.

Lastly, depending on the efficiency of the plan, it will be stored together with its level of efficiency within the beliefs base. In the paragraphs below, we give a specific example in detail.

\section{Results and conclusions}

This paper has presented an intelligent agent specifically developed to improve the medical care in geriatric residences. In regard to efficiency and improvement in the quality of the services provided by the residence, the implantation of the intelligent environment has allowed an increment of the productivity as well as providing novel additional services, such as security or automatic patient monitoring. Figure 4 shows the average number of nurses working simultaneously (each of the 24 hours of the day) at the 
residence before and after the implantation of the system prototype. As can be seen in Figure 4, the blue line represents the average number of nurses required in the residence each hour of a day without the AmI-P. The violet slides represents the same measure but after the implementation of the intelligent environment. As can be seen, the intelligent environment helps the nurses to gain time, which can be dedicated to the care of special patients, to learn or to prepare new activities. The time spent on supervision and control tasks has been reduced substantially, as well as the time spent attending false alarms, while the time for direct patient care has been increased. The system also facilitates the more flexible assignation of the working shifts at the residence; since the workers have reduced the time spent on routine tasks and can assign this time to extra activities, such as exercising the patients, learning, carrying out leisure activities or just talking with the patients or with their families. Their work is automatically monitored, as well as the patient's activities. The stored information may be analysed with knowledge discovery techniques and may help to improve the quality of life for the patients and the efficiency of the centre. The security of the centre has also been improved in three ways: the system monitors the patients and guarantees that each one of them is in the right place; secondly, only authorised personnel can gain access to the residence protected areas, and thirdly, the information is stored in a more secure way using redundancy and generating continuous backups. The access to information has been protected in order to guarantee confidentiality.

Figure 4 Number of nurses working simultaneously

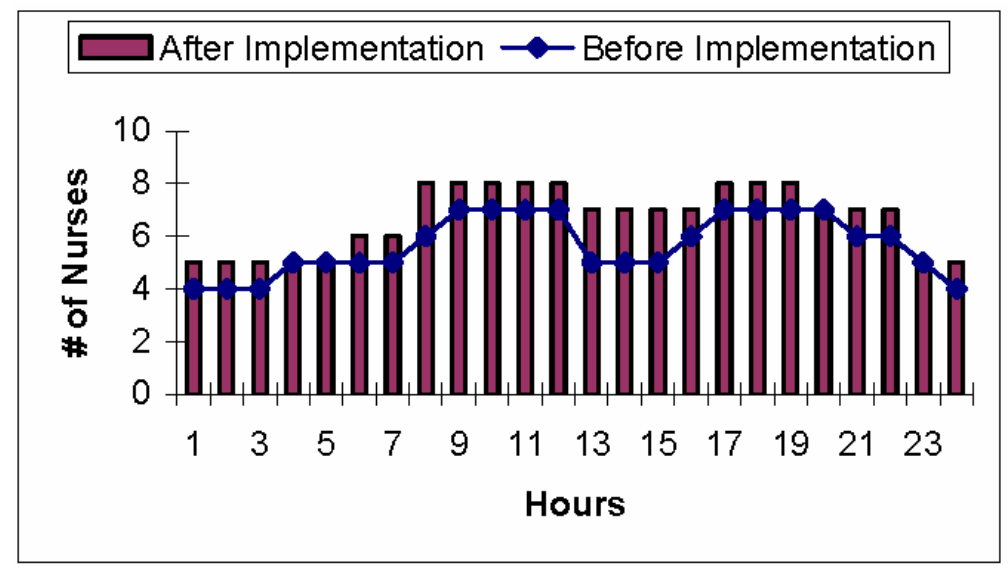

The tasks executed by nurses were divided in two categories, direct action tasks and indirect action tasks. Direct action tasks are those which require the nurse acting directly on the patient during the whole task (medication, posture change, toileting, feeding, etc.). In the indirect action tasks the nurses do not need to act directly on the patients all the time (reports, monitoring, and visits). TaskCBP planner agents can take care of some of these indirect actions, so nurses can dedicate more time to personal patients care. During the first testing period the problem was analysed and data was collected. The average time spent by nurses carrying out their duties with a given patient was obtained, having into account the patient type, its dependency level and the nurse professional level. For the direct action tasks, the following times were obtained for each patient: 35 minutes cleaning, 18 minutes feeding, eight minutes oral medication, 30 minutes parenteral 
medication, 25 minutes posture change, eight minutes toileting, 60 minutes exercise and ten minutes' others for patients with a dependence degree of one; and 45 minutes cleaning, 28 minutes feeding, 11 minutes oral medication, 42 minutes parenteral medication, 50 minutes posture change, 30 minutes toileting, 90 minutes exercise and ten minutes others for patients with a dependence degree of two. We are especially interested on time spent on indirect tasks; daily times obtained before and after the implementation. Table 2 shows how the implementation of the AmI-P reduces the time spent on indirect task. For example, the average number of minutes spent by a nurse on monitoring patients has been reduced from 167 daily minutes to 95 daily minutes without reducing the care level and the patient's safety. The novel neural network based mechanism improves the results obtained with a previous geodesic planner (Corchado et al., 2008).

Table 2 Time (minutes) spent on indirect tasks

\begin{tabular}{lccccc}
\hline & Monitoring & Reports & Visits & Other & Total \\
\hline Before & 167 & 48 & 73 & 82 & 370 \\
Geodesic planner & 105 & 40 & 45 & 60 & 250 \\
QSOR planner & 95 & 37 & 45 & 52 & 229 \\
\hline
\end{tabular}

Another interesting point taken into account to evaluate the system presented within this paper focuses on the evaluation of the novel neural network-based planning mechanism. This dynamic planning mechanism allows replanning in execution time, which in turn improves patient care. To evaluate the efficiency as well as the quality of the proposed planning mechanism, our planner has been compared to a series of different planners considered as significant in the literature for this kind of problems. This comparison can be considered as a good reference to evaluate the behaviour and benefits of the proposed replanning mechanism. The brute force technique allows obtaining a solution by exploring all the possible combinations of solutions. That is why the calculus time exponentially increases as the number of tasks increases. Another well known possibility is the use of heuristic planning techniques, as the $A^{*}$ algorithm, which allows efficient searches in the solution space, trying to reduce the number of possible combinations to explore. However, in spite of this search, the number of combinations grows exponentially (Hart et al., 1968). Once the planners used to evaluate our dynamic planner have been presented, the process followed to get an evaluation consisted on a first comparison to a brute force planner and a second comparison to a $A^{*}$ planner.

To obtain the same test conditions for the planners, the method we followed consisted of the assignment of the same tasks to nurses with the same profile and similar conditions in the environment. In order to observe the efficiency of the planners looking at the number of tasks assigned to the nurse, the number of tasks was changed. The limits for the number of tasks assigned to a nurse were established between ten and 30 . The results obtained can be observed in Table 3. As can be seen in Table 3 the times obtained for the brute force planner are estimated from 15 points up since the calculus implies excessive and impracticable computation times. Something similar happens for the $A^{*}$ algorithm based planner. The estimations have been done taking into account the number of combinations required as well as the time employed as a function of the length of the route. The number of total combinations for this problem is obtained as $N$ ! And the time employed is obtained as a comparison to the time employed for the estimation of the aptitude for a plan composed of ten tasks. In the case of the $A^{*}$ planner the results 
correspond to the estimation for an intermediate situation, in which it is only necessary to spread out the middle of the branches of each of the nodes. Basically, to implement this algorithm we need to define an evaluation function in the way $f^{*}(n)=g^{*}(n)+h^{*}(n)$, where $g^{*}(n)$ is the minimum cost to go from the origin node to the node $\mathrm{n}$ and $h^{*}(n)$ is the minimum cost to reach the final node from the node $n$. As these functions are unknown, we need to estimate them by means of $f(n)=g(n)+h(n)$ ( $A$ algorithm), imposing the restriction $h(n) \leq h^{*}(n) . H(n)$ has been defined as $h(n)=0$ and $g(n)$ as the value for the accumulated route following the branches of the tree. Initially we begin from a state without any task assigned, so in the next level we are going to add so many branches as tasks have to be added. After this, the distances for the routes are calculated, as well as the fulfilment of the time restrictions and the valid branch with a minor distance $f(n)$ is chosen.

As can be seen in the results shown in Table 3, the TaskCBP planner agent has a better behaviour with respect to the increment of the number of tasks than the brute force and $A^{*}$ planner. The distances calculated, although not optimum, are very close to the result, so they can be considered as acceptable. The other planners are impracticable for 20 tasks up. That's the reason why it is impossible to replan in execution time for a number of tasks higher to 15 elements.

Table 3 Planner comparison

\begin{tabular}{lcccccc}
\hline $\begin{array}{l}\text { Number } \\
\text { of task }\end{array}$ & $\begin{array}{c}\text { Brute force } \\
\text { time }(s)\end{array}$ & Distance & $\begin{array}{c}A^{*} \text { algorithm } \\
\text { time }\end{array}$ & Distance & \multicolumn{2}{c}{ TaskCBP } \\
\hline 10 & 237.63 & 878 & 0.01572 & 878 & 4.32 & 878 \\
15 & $85,652,671.1$ & $\ldots$ & 3.3012 & 1,573 & 6.12 & 1,590 \\
20 & $1.5936 \mathrm{E}+14$ & $\ldots$ & 237.6864 & 1,857 & 8.35 & 1,897 \\
25 & $1.016 \mathrm{E}+21$ & $\ldots$ & $313,746.048$ & $\ldots$ & 9.79 & 2,551 \\
30 & $1.7374 \mathrm{E}+28$ & $\ldots$ & $57,101,780.7$ & $\ldots$ & 12.11 & 2,856 \\
\hline
\end{tabular}

The CBP planning mechanism which allows replanning in execution time, integrated within an intelligent agent, has been tested over the last few months. To test the system 30 patient agents, ten nurse agents, two doctor agents and one manager agent were instantiated. During the testing period the systems usefulness has been evaluated from different points of view. The system, which in turn improves patient care, facilitates the more flexible assignation of the working shifts at the residence; since the workers have reduced the time spent on routine tasks and can assign this time to extra activities, such as exercising the patients, learning, carrying out leisure activities or just talking with the patients or with their families. Their work is automatically monitored, as well as the patients' activities. The stored information may be analysed with knowledge discovery techniques and may help to improve the quality of life for the patients and the efficiency of the centre. The security of the centre has also been improved in three ways: the system monitors the patients and guarantees that each one of them is in the right place; secondly, only authorised personnel can gain access to the residence protected areas, and thirdly, the information is stored in a more secure way using redundancy and generating continuous backups. The access to information has been protected in order to guarantee confidentiality.

From the point of view of ambient intelligence (Ducatel et al., 2001; Friedewald and $\mathrm{Da}$ Costa, 2003), the present work evaluates the capabilities of ubiquitous 
computing, ubiquitous communication and intelligent user interaction that the proposed system presents. The system facilitates carrying out tasks in a ubiquitous way, such as plans generation for nurse's working day, or patient and task assignation. These tasks are executed in a distributed way inside the intelligent environment and are independent of the location of the processing devices due to the use of mobile devices and wireless networks. In a similar manner, the system provides wireless communication by means of WiFi and RFID technologies, allowing location-independent access. Finally, the system incorporates intuitive interfaces which facilitate usability and automatic adaptation to the user profile. We had certain problems implementing the system, partly because the nurses and workers were not familiar with the use of PDA devices, so some courses were given to introduce them to these technologies and teach them how to use the system interface. After that and with some difficulties with the installation of the wireless access points (with the propagation of the signal) and the collocation of the RFID door readers, the system was running smoothly, with only minor problems.

The agent technology provides a framework for distributed problem solving and data communication. The characteristics of agents, such as autonomy, reactivity, pro-activity, social abilities, reasoning, learning and mobility (Wooldridge and Jennings, 2005) are adequate to fulfil the AmI necessities. Moreover, the incorporation of CBR mechanisms (Aamodt and Plaza, 1994) facilitates ubiquitous computation capabilities. An agent can act as an interface between the user and the environment. In this way, it is possible to get that the services provided by the multiagent system and the technology in the environment can be adapted to the user necessities. Finally, agents can be executed on mobile devices, which facilitate ubiquitous capabilities. These characteristics make the proposed architecture appropriate for developing AmI (Corchado et al., 2008) scenarios. The manager agent assigns tasks and monitors the patients and nurses locations. Nurse and doctor agents run on handheld devices and provide easy-touse interfaces.

In the future, healthcare for residence patients, the elderly and people with other disabilities will require the use of new technologies that allow medical personnel to carry out their tasks more efficiently. Weick (1985) describes the fundamental problems of knowledge transfer and sense making in digital/computer based environments. We have shown the potential of deliberative TaskCBP agents in a distributed multi-agent system focused on healthcare, providing a way to respond to some challenges of healthcare, related, e.g., to the identification, control and healthcare planning. In addition, the use of RFID technology on people provides a high level of interaction among users and patients through the system and is fundamental in the construction of the intelligent environment. Furthermore, the use of mobile devices, when used well, can facilitate social interactions and knowledge transfer.

\section{Acknowledgements}

This work has been partially supported by the Spanish Ministry of Science and Technology project TIN2006-14630-C03-03 and the Spanish Ministry of Labor and Social Security project 'Plataforma inteligente para la gestión integral de residencia geriátricas'. Special thanks to Sokymat by the RFID technology provided and to Telefónica Móviles (Movistar) for the wireless devices donated. 


\section{References}

Aamodt, A. and Plaza, E. (1994) 'Case-based reasoning: foundational issues, methodological variations, and system approaches', AI Communications, Vol. 7, pp.39-59.

Angulo, C. and Tellez, R. (2004) 'Distributed intelligence for smart home appliances', Tendencies of Data Mining in Spain, Spanish Data Mining Network, Barcelona, Spain.

Bajo, J., de Paz, Y., de Paz, J.F., Martín, Q. and Corchado, J.M. (2006) 'SMas: a shopping mall multiagent systems', Proceedings of IDEAL'06, LNAI, Springer Verlag, Vol. 4224, pp.166-1173.

Bajo, J., Julián, V., Corchado, J.M., Carrascosa, C., de Paz, Y., Botti, V. and de Paz, J.F. (2008) 'An execution time planner for the ARTIS agent architecture', Engineering Applications of Artificial Intelligence, in Press.

Bergmann, R., Kolodner, J. and Plaza, E. (2005) 'Representation in case-based reasoning', The Knowledge Engineering Review, Vol. 00, No. 0, pp.1-4, Cambridge University Press.

Camarinha-Matos, L. and Afsarmanesh, H. (2002) 'Design of a virtual community infrastructure for elderly care', Camarinha-Matos, L.M. (Ed.): Proceedings of PRO-VE'02, Sesimbra, Portugal.

Camarinha-Matos, L., Rosas, J. and Oliveira, A. (2004) 'A mobile agents platform for telecare and teleassistance', Proceedings of TELECARE 2004, Porto, Portugal.

Cesta, A., Bahadori, S., Cortellesa, G., Grisetti, G., Giuliani, M., Locchi, L., Leone, G., Nardo, D., Oddi, A., Pecora, F., Rasconi, R., Saggese, A. and Scopelliti, M. (2003) 'The RoboCare project, cognitive systems for the care of the elderly', Proceedings of ICADI'03, Washington DC, USA.

Corchado, J.M. and Laza, R. (2003) 'Constructing deliberative agents with case-based reasoning technology', International Journal of Intelligent Systems, Vol. 18, pp.1227-1241.

Corchado, J.M., Pavón, J., Corchado, E.S. and Castillo, L.F. (2005) 'Development of CBR-BDI agents: a tourist guide application', Proceedings of ECCBR'04, LNAI, Springer Verlag, Vol. 3155, pp.547-559.

Corchado, J.M., Bajo, J. and Tapia, D.I. (2006) 'ALZ-MAS: Alzheimer's special care multi-agent system', Proceedings of the Workshop on Health Care ECAI'06, pp.1-9, Riba del Garda, Italy.

Corchado, J.M., Aiken, J. and Bajo, J. (2007) 'A CBP agent for monitoring the CO2 exchange rate', Series on Computacional Science, Springer Verlag, Vol. 73, pp.213-246.

Corchado, J.M., Bajo, J., de Paz, Y. and Tapia, D.I. (2008) 'Intelligent environment for monitoring Alzheimer patients, agent technology for health care', Decision Support Systems, Eslevier Science, Vol. 44, No. 2, pp.382-396.

Cox, M.T., Muñoz-Avila, H. and Bergmann, R. (2006) 'Case-based planning', Knowledge Engineering Review, Vol. 20, No. 3, pp.283-287.

Ducatel, K., Bogdanowicz, M., Scapolo, F., Leijten, J. and Burgelman, J.C. (2001) 'That's what friends are for ambient intelligence (AmI) and the IS in 2010', Innovations for an E-Society.

Emiliani, P.L. and Stephanidis, C. (2005) 'Universal access to ambient intelligence environments: opportunities and challenges for people with disabilities', IBM Systems Journal.

Friedewald, M. and Da Costa, O. (2003) 'Science and technology roadmapping: ambient intelligence in everyday life (AmI@Life)', Institute for Prospective Technology Studies IPTS, Working paper, Sevilla.

Glez-Bedia, M. and Corchado, J.M. (2002) 'A planning strategy based on variational calculus for deliberative agents', Computing and Information Systems Journal, Vol. 10, No. 1, pp.2-14.

Hart, P.E., Nilsson, N.J. and Raphael, B. (1968) 'A formal basis for the heuristic determination of minimum cost paths graphs', IEEE Trans. on Systems Science and Cybernetics, Vol. SSC-4, No. 2, pp.100-107. 
Jin, H.D., Leung, K.S., Wong, M.L. and Xu, Z.B. (2003) 'An efficient self-organizing map designed by genetic algorithms for the traveling salesman problem', IEEE Transactions on Systems, Man, and Cybernetics Part B: Cybernetics, Vol. 33, No. 6, pp.877-888.

Kohonen, T. (1984) Self-Organization and Associative Memory, Springer Verlag.

Kolodner, J. (1993) Case-Based Reasoning, Morgan Kauffman, San Mateo.

Leung, K.S., Jin, H.D. and Xu, Z.B. (2004) 'An expanding self-organizing neural network for the traveling salesman problem', Neurocomputing, Vol. 62, pp.267-292.

Richter, K. and Hellenschmidt, M. (2004) 'Interacting with the ambience: multimodal interaction and ambient intelligence', Proceedings of the W3C Workshop on Multimodal Interaction, pp.19-20, Shophia Antipolis, France.

US Department of Commerce (2005) 'Radio frequency identification: opportunities and challenges in implementation', Technical report.

Watson, I. (1997) Applying Case-Based Reasoning: Techniques for Enterprise Systems, Morgan Kaufmann Publishers Inc., San Francisco, CA, USA.

Weick, K.E. (1985) 'Cosmos vs. chaos: sense and nonsense in electronic contexts', Ruggles, R.L. (Ed.): Knowledge Management Tools, Vol. 3.

Wooldridge, M. and Jennings, N.R. (Eds.) (1995) 'Agent theories, architectures, and languages: a survey’, Intelligent Agents, Springer-Verlag, pp.1-22. 